\title{
Modelling of heat and mass transfer processes in phase transformation cycle of sprayed water into gas: 4. Thermal state analysis of a droplet slipping in humid air flow
}

\author{
G. Miliauskas*, M. Maziukienė**, A. Balčius***, J. Gudzinskas**** \\ Kaunas University of Technology, Studentu 56, LT-51424 Kaunas, Lithuania \\ E-mail:*gimil@ktu.lt,**monika.maziukiene@ktu.lt,***algimantas.balcius@ktu.lt,****juozas.gudzinskas@ktu.lt
}

cross $^{\text {ref }}$ http://dx.doi.org/10.5755/j01.mech.22.2.13314

\section{Nomenclature}

$a$ - thermal diffusivity, $\mathrm{m}^{2} / \mathrm{s} ; c_{p}$ - mass specific heat, $\mathrm{J} /(\mathrm{kg} \mathrm{K}) ; F_{O}$ - Fourier number; $g$ - evaporation velocity, $\mathrm{kg} / \mathrm{s} ; L$ - latent heat of evaporation, $\mathrm{J} / \mathrm{kg} ; m$ - vapour mass flux, $\mathrm{kg} /\left(\mathrm{m}^{2} \mathrm{~s}\right) ; p$ - pressure, $\mathrm{Pa} ; q$-heat flux, $\mathrm{W} / \mathrm{m}^{2}$; $r$-radial coordinate, $\mathrm{m} ; \quad P e$-Peclet number; $T$ - temperature, $\mathrm{K} ; \lambda$-thermal conductivity, $\mathrm{W} /(\mathrm{m} \mathrm{K})$; $\mu$ - molecular mass, $\mathrm{kg} / \mathrm{kmol} ; \rho$-density, $\mathrm{kg} / \mathrm{m}^{3} ; \tau$ - time; $w$ - velocity, $\mathrm{m} / \mathrm{s}$;

subscripts -

$C$-droplet centre; co-condensation; $c$ - convection; $e$ - evaporation; $f$ - phase change; $g$ - gas; $i$ - time index in a digital scheme; it - number of iteration; $I$ - index of control time; $j$-index of radial coordinate; $J$-index of droplet surface; $k$-conduction; $k+r$-conduction and radiation; $l$-liquid; $m$-mass average; $\eta$-non-dimensional radial coordinate; $r$-radiation; $R$-droplet surface; $v$-vapor; $v g$-gas-vapor mixture; 0 -initial state; $\infty$-far from a droplet;

superscripts -

+ - external side of a droplet surface; - - internal side of a droplet surface.

\section{Introduction}

Hot gases that are produced in organic solid gasification process is effectively cooled down by water injection into transportation systems, conditioned air, combustion process is controlled and eliminated fumes parameters. For mention-above and other water sprayed application cases thermal energy consumptions is important for proceeding phase transformations on the droplet surface and for liquid heating in the droplet. Therefore researches of water droplets heat transfer and evaporation [1-7] as well vapour condensation on its surface [8-12] is relevant.

The change of gas mixture composition that carry out droplets is caused by ongoing phase transitions on the surface of the droplet and temperature variations are defined by droplet heat transfer processes. Heat flow that is provided by the gas stimulates a liquid evaporation from droplet surface and also warms liquid in a droplet. Liquid warming rate is defined by intensity of heat offtake to droplet. In combined heating case the heat is being provided by convection and radiation: $q_{\Sigma}^{+}=q_{c}^{+}+q_{r}^{+}$. Heat supplying for droplets by convection and radiation has its own peculiarities that is defined by transfer process nature. A flowing round fluid provides heat for the surface by convection, while at semi-transparent droplet a radiation heat is provided by absorbing electromagnetic waves of light in the infrared spectrum. Therefore, the radiation heat warmth the liquid in the droplet directly, and external convection heat from a droplet surface must be leaded off inside the droplet by internal heat exchange. Therefore, a total heat flow density of a droplet $q_{\Sigma}^{-}=q_{c}^{-}+q_{r}^{-}$defines a fluid heating intensity. Semi-transparent liquids absorbs spectral radiation by surface poorly [6], so assumption $q_{r}^{-} \equiv q_{r}^{-}$is popular. A convective component $q_{c}^{-}$of total heat flow is defined by droplet circulation and heat conductivity processes. Internal layers of a droplet are heated unevenly, therefore a droplet thermal state is described by variable function $T(x, y, z, \tau)$ in time and space. A droplet unsteady temperature field is described by time and radial coordinate function $T(r, \tau)$ in spherically symmetric heating assumption case. A thermal state of non-isothermal droplet is defined by mass average temperature function of a droplet:

$$
T_{l, m}(\tau)=\frac{\int_{0}^{R(\tau)} r^{3} \rho_{l}(r, \tau) T_{l}(r, \tau) d r}{\int_{0}^{R(\tau)} r^{3} \rho_{l}(r, \tau) d r} .
$$

A droplet heating conditions in gas flow [10], has a strong impact for peculiarities of droplet unsteady temperature function $T(r, \tau)$. When external heating provides a heat spread by radiation and conductivity, function $T(r, \tau)$ is defined according to integral type model [7] that is convenient for numerical modelling. Then heat that is leaded off to the droplet is described by Fourier's low. In case of convective heating droplet slips in gas flow and friction forces rises on droplet surface. On their impact liquid is circulating and intensifies internal heat transfer. In case of forced liquid circulation at the droplet its thermal state is described by energy and Navier-Stokes equation system. The latter analytical solution is not possible and the numerical solution is complicated [12]. Therefore a numerical scheme of convection heat flow in a droplet would be susceptible for machine computing and would complicate iterative method application whereby a droplet surface temperature definition is based on. To define unsteady temperature field function $T_{l}(\eta, \tau) \equiv T_{l,}, c^{\prime}(\eta, \tau)$ in case of slipping droplet is quite difficult.

In this work a slipping droplet thermal state defining methodology is developed and slipping intensity impact for water droplet warming in humid air flow is also analysed. 


\section{Research method and results}

For droplet thermal state definition the onedimensional effective conductivity empirical model is provided [13] and heat transfer by conduction and radiation in the droplet integral models [7]. To define a droplet surface temperature time function $T_{R}(\tau)$ a balance of fluxes that flows in and off on the surface of a droplet is provided. This is defined by expressions (2) and (3) for condensing and evaporation regimes, respectively.

$$
\begin{aligned}
& q_{c}^{-}(\tau)=q_{c}^{+}(\tau)+q_{f \equiv c o}^{+}(\tau) ; \\
& q_{c}^{-}(\tau)=q_{c}^{+}(\tau)-q_{f \equiv e}^{+}(\tau) .
\end{aligned}
$$

At expressions (2) and (3) a potential radiation flow absorption by droplet surface is denied. External convective heat flow is described by the method [10,13]. Phase transformation flow on the surface of the droplet is described by water vapour flow density $q_{f}^{+}=\left|m_{v}^{+}\right| L$, while for convective het flow description that is leaded to droplet a modified Fourier low is applied:

$$
q_{c}^{-}(\tau)=-\left.k_{c}^{-}(\tau) \lambda_{l}(\tau) \frac{\partial T_{l}(r, \tau)}{\partial r}\right|_{r \equiv R} .
$$

At compound heating case by conductivity and radiation an unsteady temperature field gradient is described by infinite integral equations series [7]:

$$
\begin{aligned}
& \left.\frac{\partial T_{l}(r, \tau)}{\partial r}\right|_{r=R}=\sum_{n=1}^{\infty} \frac{2}{R^{2}} \int_{0}^{\tau}\left[R \frac{d T_{R}}{d \tau}+\frac{(-1)^{n} n \pi}{R \rho_{l} c_{p, l}} \times\right. \\
& \times \int_{0}^{R}\left(\sin \frac{n \pi r_{*}}{R}-\frac{n \pi r_{*}}{R} \cos \frac{n \pi r_{*}}{R}\right) q_{r} d r_{*} \times \\
& \times \exp \left[-\frac{a_{l}(n \pi)^{2}}{R^{2}}\left(\tau-\tau_{*}\right)\right] d \tau_{*} .
\end{aligned}
$$

Liquid circulation potential input to balance of heat spread in a droplet is evaluated by effective heat conductivity parameter where at expression (6) Peclet number is defined according to liquid flow maximum velocity $w_{l, R}$ : $P e_{l}=2 R \rho_{l} w_{l, R} / \mu_{l}[13]$ on the surface of a droplet. This flow is caused by friction forces.

$$
k_{c}^{-}(\tau)=1.86+0.86 \tanh \left[2.245 \lg \frac{P e_{l}(\tau)}{30}\right] .
$$

Iterative numerical scheme is made from expanded (2) and (3) expressions. A droplet surface time function $T_{R}(\tau)$ definition of this scheme is applicable for all droplet heating cases. However, it must be remember, that surface temperature function $T_{R}(\tau)$ defines only marginal value of unsteady temperature field $T(r, F o)$ function of a droplet $T(r \equiv R, F o)=T_{R}(F o)$. Therefore, a local temperature $T(r<R, F o)$ according to discussed methodology can be defined only at assumption of stable liquid at the droplet. In humid air slipping water droplet phase transformation cycle is modelled according to boundary conditions $T_{g}=500 \mathrm{~K}, p=0.1 \mathrm{MPa}, R_{0}=0.000075 \mathrm{~m}, T_{l, 0}=278 \mathrm{~K}$, $\bar{p}_{v, \infty}=0.3$ and analysed in Fourier time scale $F o=\tau a / R_{0}^{2}$. Primary droplet slipping in humid air has a significant impact for calculated thermal conductivity parameter that defines water circulation intensity in a droplet (Fig. 1).

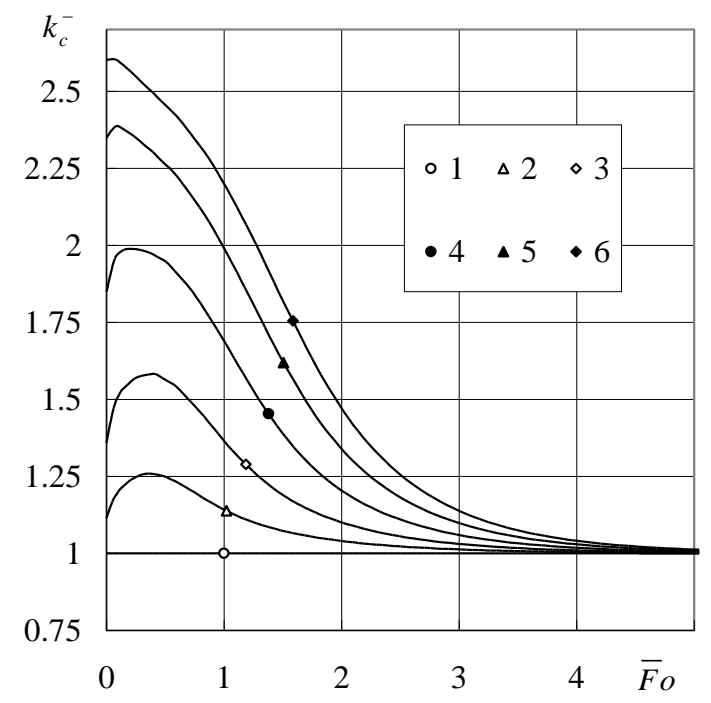

Fig. 1 Droplet primary slipping in air flow impact for effective thermal conductivity parameter. $R e_{0}: 1-0$, $2-5,3-10,4-20,5-40,6-80 ; \bar{F} o=F o / F o_{c o, " k "}$

A point that defines Reynolds number initial value (Fig. 1), also shows phase transformations of condensing regime relative duration in aspect of droplet heating by conduction. It is clearly seen, that condensation regime time increases and convectional heat transformation at the droplet is more prevalent if droplet slips more intensively. Water circulation intensity is sensitive for droplet slipping and dispersity. A droplet growth in condensation regime is suitable factor for circulation in it, while droplet slipping weakening is factor that reduces water circulation in droplet. At the beginning of condensing regime an intensification of convectional heat transfer observes (Fig. 1). This means, that for some time, a droplet growth factor that is favourable for droplet circulation is stronger than slipping weakening factor that repress circulation. At both factors balancing point $k_{c}^{-}(F o)$ extreme point observes in function graphs (Fig. 1), after which the water circulation consistently suffocates in the droplet.

The droplet initial slipping significantly influences the droplet surface warming process (Fig. 2). The droplet initial slipping significantly influences the droplet surface warming process (Fig. 2). In unsteady phase transformation regime a droplet surface heats up rapidly (Fig. 2, a), however warming rate consistently suffocates until becomes zero at final stage of unsteady evaporation (Fig. 3, a). In equilibrium evaporation regime a temperature of droplet heating by conductivity is steady. A slipping droplet temperature decreases at equilibrium evaporation regime (Fig. 2, b): at the beginning of equilibrium evaporation droplet cooling down even accelerates, however it rapidly reaches maximum cooling down rate and after that is consistently weakening (Fig. 3, b). Therefore, at equilibrium evaporation process when droplet slipping reduces approaching to thermal state of droplet heated by conductivity is getting closer (Fig. 2, b). Deeper inner lay- 
ers heating process analysis is required to define slipping droplet thermal state. A droplet slipping causes a convection " $c$ " heat transfer in therein. Its intensity is defined by local heat flow density function $q_{c}^{-}(r, \tau)$.
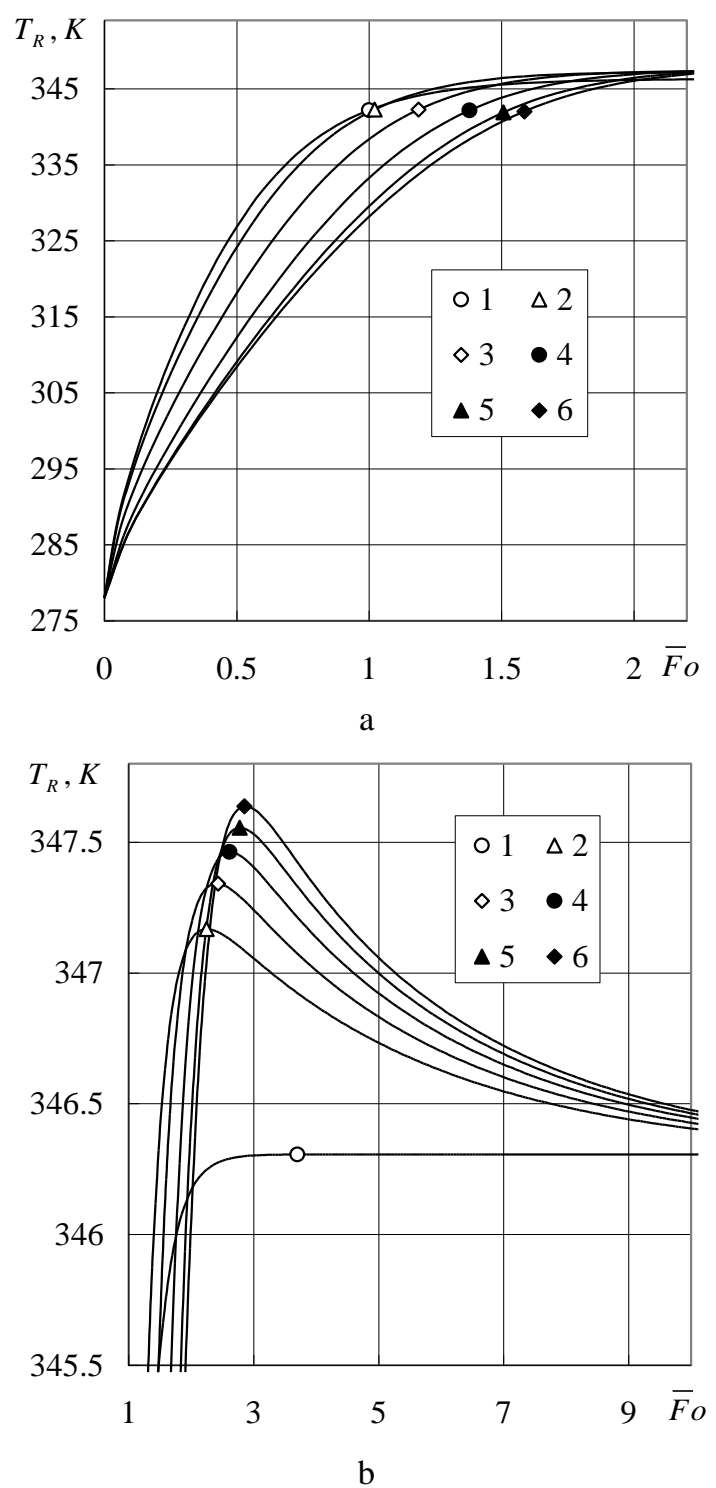

Fig. 2 Droplet primary slipping in air flow impact for surface temperature: a - unsteady phase transformation regime; $\mathrm{b}$ - unsteady evaporation final and equilibrium evaporation primary states. $R e_{0}: 1-0,2-5,3-$ $10,4-20,5-40,6-80$

The latter is difficult to describe due to fact that droplet diameter is changing at phase transformation cycle. Therefore a dimensionless radial coordinate $\eta=r / R(F o)$ of droplet is introduced. By its aspect a droplet dimensionless radius is universal, because its unit value remains in all droplet phase transformation cycle $F o \equiv 0 \div F o_{c o} \div F o_{n f} \div F o_{f}$. It is convenient for droplet unsteady temperature field $T_{\eta, "}{ },(F o)$, local gradient $\operatorname{grad}_{\eta, "},(F o)$ and local convectional heat flow density $q_{\eta, " c "}^{-}(F o)$ functions description. It is assumed that function $F_{\eta, "{ }^{\prime \prime}}^{-}(F o)$ defined by effective thermal conductivity local parameters is existing. Effective thermal heating conductivity theory is applied for convective transfer intensity description inside droplet:

$$
q_{\eta, " c "}^{-}(F o)=-F_{\eta, " c "}^{-}(F o) \cdot \lambda_{\eta}(F o) \cdot \operatorname{grad} T_{\eta, " k "}(F o) .
$$

Local temperature gradient is described by integral equation:

$$
\begin{aligned}
& \frac{\partial T_{l}(r, \tau)}{\partial r}=2 \sum_{n=1}^{\infty}\left(\frac{n \pi}{r R} \cos \frac{n \pi r}{R}-\frac{1}{r^{2}} \frac{n \pi r}{R}\right) \times \\
& \times \int_{0}^{\tau}\left[\frac{(-1)^{n} R}{n \pi} \frac{d T_{R}}{d \tau} \exp \left[-\frac{a_{l}(n \pi)^{2}}{R^{2}}\left(\tau-\tau_{*}\right)\right] d \tau_{*} .\right.
\end{aligned}
$$

Condition $r \equiv R$ is applied for expression (8) and temperature gradient is defined for leaded off convectional heat flow description at the droplet (4) in expression (Fig. 4).
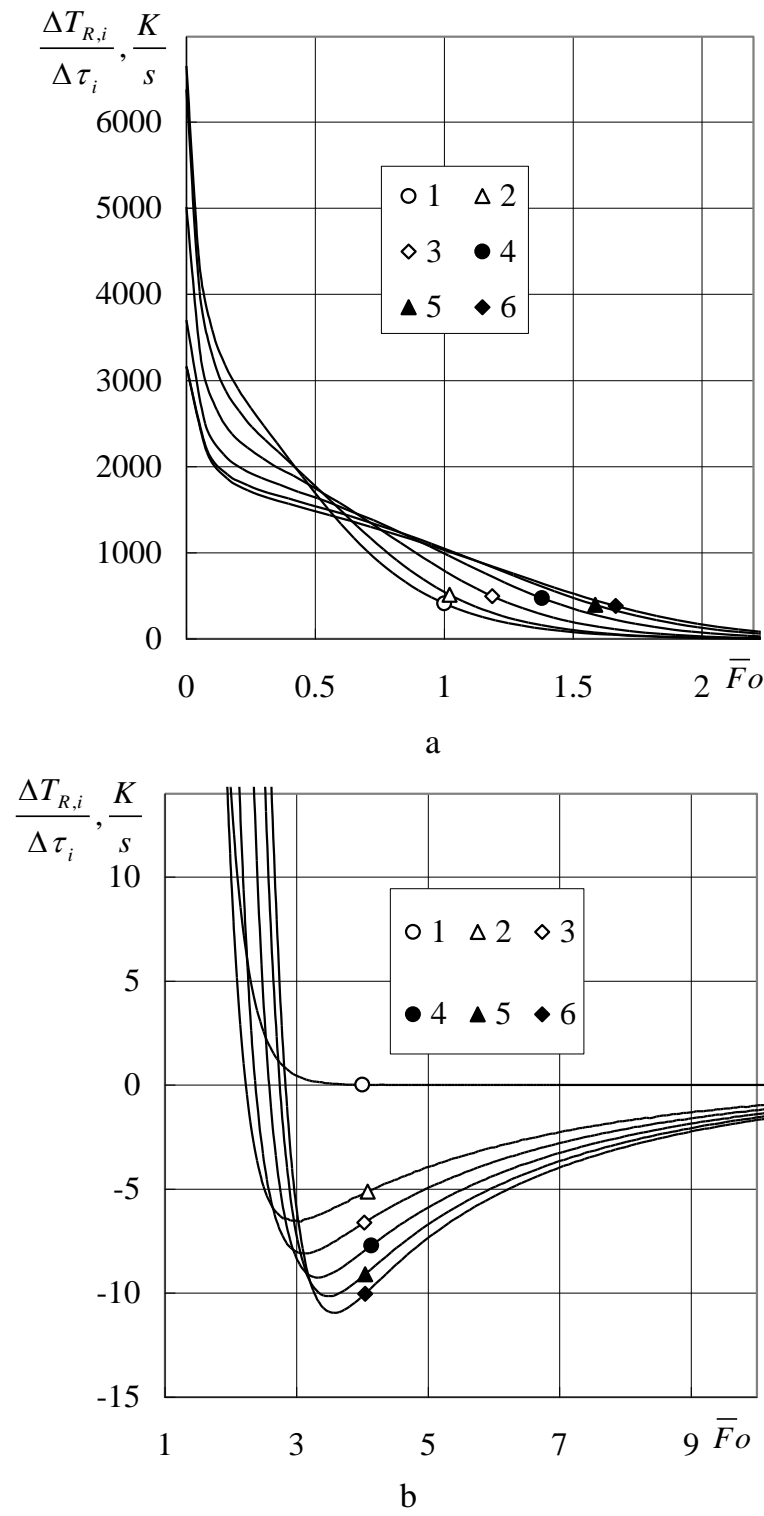

Fig. 3 Droplet primary slipping in air flow impact for its surface warming rate: a-unsteady phase transformation regime; b-unsteady evaporation final and equilibrium evaporation primary states. $R e_{0}: \quad 1-0,2-5,3-10,4-20,5-40,6-80$; $\Delta \bar{T}_{R, i}=\left(T_{R, i+1}-T_{R, i}\right) / T_{l, 0} ; \Delta F o_{i}=F o_{i+1}-F o_{i}$ 
A convectional heat local flow density function is composite $q_{\eta, " c}^{-}(F o)$. It is created from components of convective $q_{\eta, c, c^{\prime \prime}}^{-}(F o)$ that is caused by circulation and conductive $q_{\eta, k, " c "}^{-}(F o)$ that is defined by heat conductivity fluid:

$$
q_{\eta, " c "}^{-}(F O)=q_{\eta, c, " c "}^{-}(F O)+q_{\eta, k, " c "}^{-}(F O) .
$$

Heat conductivity component of a droplet is defined by heat conductivity Fourier low at (9) expression:

$$
q_{\eta, k, c^{\prime \prime}}^{-}(F o)=-\lambda_{\eta}(F o) \cdot \operatorname{grad} T_{\eta, c^{\prime \prime}}(F o) .
$$

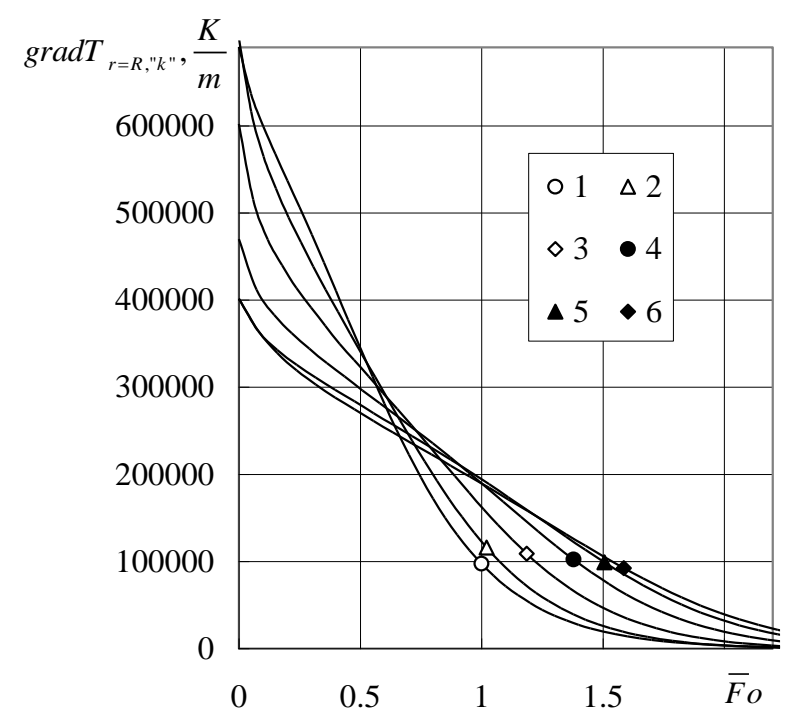

Fig. 4 Droplet primary slipping in air flow impact for temperature gradient that is calculated according to stable liquid model inside the droplet. $R e_{0}: 1-0,2-5$, $3-10,4-20,5-40,6-80$

Convection heat flow component that is caused by liquid circulation in the droplet can be defined according to difference of local convectional heat flow and its conductivity component:

$$
\begin{aligned}
& q_{\eta, c, " c^{\prime \prime}}^{-}(F o)=q_{\eta, " c^{\prime \prime}}^{-}(F o)-q_{\eta, k, " c^{\prime \prime}}^{-}(F o)=-F_{\eta, " c^{\prime \prime}}^{-}(F o) \times \\
& \quad \times \lambda_{\eta}(F o) \cdot \operatorname{grad} T_{\eta, " k "}(F o)+\lambda_{\eta}(F o) \cdot \operatorname{grad} T_{\eta, " c^{\prime \prime}}(F o) .(11)
\end{aligned}
$$

It is assumed that at unsteady temperature field in local gradient " $k$ " and " $c$ " heat exchange cases a proportion of the effective thermal conductivity is:

$$
\frac{\operatorname{grad} T_{\eta, " k "}(F o)}{\operatorname{grad} T_{\eta, " c "}(F o)} \equiv F_{\eta, " c "}^{-}(F o) .
$$

Then convectional heat flow component that is caused by liquid circulation in the droplet is defined according to expression:

$$
q_{\eta, c, " c^{\prime \prime}}^{-}(F o)=\left[F_{\eta, " c^{\prime \prime}}^{-}(F o)-\frac{1}{F_{\eta, c^{\prime \prime}}^{-}(F o)}\right] q_{\eta, " k^{\prime \prime}}(F o) .
$$

In modelled phase transformation cycle a time change step $\Delta F o_{i} \equiv F o_{I} /(I-1)$ is defined by providing a finite number of control points in free chosen Fourier number changing interval $F o \equiv 0 \div F o_{I}$. Providing a finite $J$ number of control points a unit radius of a droplet is divided to $J$-1 interval $\Delta \eta_{j} \equiv 1 /(J-1)$. Preserved conditions:

$$
\left.\begin{array}{l}
\sum_{i=1}^{I-1} \Delta F o_{i} \equiv \sum_{i=1}^{I-1}\left(F o_{i+1}-F o_{i}\right)=F o_{k o n} \\
\sum_{j=1}^{J-1} \Delta \eta_{j} \equiv \sum_{j=1}^{J-1}\left(\eta_{j+1}-\eta_{j}\right)=1 .
\end{array}\right\}
$$

When defining temperature field gradient in droplet by temperature difference and radial coordinate change ratio $\operatorname{grad} T_{j, i,{ }^{\prime \prime} c^{\prime \prime}} \equiv\left(T_{j+1, i,{ }^{\prime \prime} c^{\prime \prime}}-T_{j, i,{ }^{\prime \prime} c^{\prime \prime}}\right) /\left(\eta_{j+1}-\eta_{j}\right)$, a droplet local temperature when water circulates in it, can be defined by scheme:

$$
\begin{aligned}
& T_{j, i, " c^{\prime \prime}}=T_{J, i,{ }^{\prime \prime} c^{\prime \prime}}-R_{i} \times \\
& \times \sum_{j}^{J-1} \frac{\operatorname{grad} T_{j, i, c^{\prime \prime}}+\operatorname{grad} T_{j+1, i, c^{\prime \prime}}}{2}\left(\eta_{j+1}-\eta_{j}\right) .
\end{aligned}
$$

A strong and liner receding circulation cases in a droplet is provided for parameter function $F_{\eta, " c "}^{-}(F o)$ definition. In case of strong circulation:

$$
F_{\eta_{j, i, c^{\prime \prime}}^{-}}^{-} \equiv k_{c, i}^{-}
$$

At case of liner receding water circulation inside the droplet the function $F_{\eta, c^{\prime \prime}}^{-}(F o)$ is defined according to scheme:

$$
F_{\eta_{j, i, c^{\prime \prime}}}^{-}=1+\frac{j-1}{J-1} \cdot\left(k_{c, i}^{-}-1\right) .
$$

A Modelled droplet slipping regimes in air flow is defined by freely chosen Reynolds number $R e_{0}$ at interval $R e_{0} \equiv 0 \div 100$. At each case $I=21$ and $J=41$, while Fourier criteria $F_{O_{I}}$ is equated for criterion $F_{o_{c o}}$ that defines phase transformation regime duration $F o_{I}=F o_{c o}\left(R e_{0}\right)$ [17]. Up to 225 Fourier time steps $\Delta F o_{i}$ is made in phase transformation cycle.

Table 1

Heat flow densitys on the surface of a droplet, $\mathrm{kW} / \mathrm{m}^{2}$

\begin{tabular}{|c|c|c|c|c|c|c|}
\hline $\operatorname{Re}_{0}$ & $q_{c, 0}^{+}$ & $q_{f, 0}^{+}$ & $q_{c, 0}^{-}$ & $\begin{array}{c}q_{c, c o}^{-} ; \\
q_{c, c o}^{+}\end{array}$ & $\begin{array}{c}q_{f, c o}^{+} ; \\
q_{c, n f}^{-}\end{array}$ & $\begin{array}{c}q_{f, n f}^{+} \\
q_{c, n f}^{+}\end{array}$ \\
\hline 0 & 105.8 & 282.2 & 388 & 62.7 & 0 & 61.5 \\
\hline 5 & 156.6 & 282.2 & 439.4 & 87.6 & 0 & 74.5 \\
\hline 10 & 177.4 & 282.2 & 460.2 & 92.8 & 0 & 77.1 \\
\hline 20 & 206.5 & 282.2 & 489.3 & 96.1 & 0 & 78.5 \\
\hline 40 & 247.4 & 282.2 & 530.2 & 100.2 & 0 & 79.8 \\
\hline 80 & 305.1 & 282.2 & 587.9 & 103.9 & 0 & 81.6 \\
\hline
\end{tabular}

A droplet energy state is defined by external convective heat $Q_{c}^{+}$, phase transformation heat $Q_{f}^{+}$and internal convective heat $Q_{c}^{-}$flows. Their calculated densities 
$q_{c}^{+}, q_{f}^{+}$and $q_{c}^{-}$at the beginning of phase transformation regime and condensing regime change to evaporation regime as well at equilibrium evaporation beginning moments for different $\mathrm{Re}_{0}$ values is given in Table 1 .

Internal convection heat flow $Q_{c}^{-}$spreads by heat conductivity and is transferred by circulating water in droplet. Therefore internal heat flow intensity is defined by (10) and (13) expression which is described by conduction and convection heat flow components $q_{c}^{-}=q_{\eta=1, k, " c^{\prime \prime}}^{-}+q_{\eta=1, c, " c "}^{-}$. Internal convection heat flow density components are given in Table 2 .
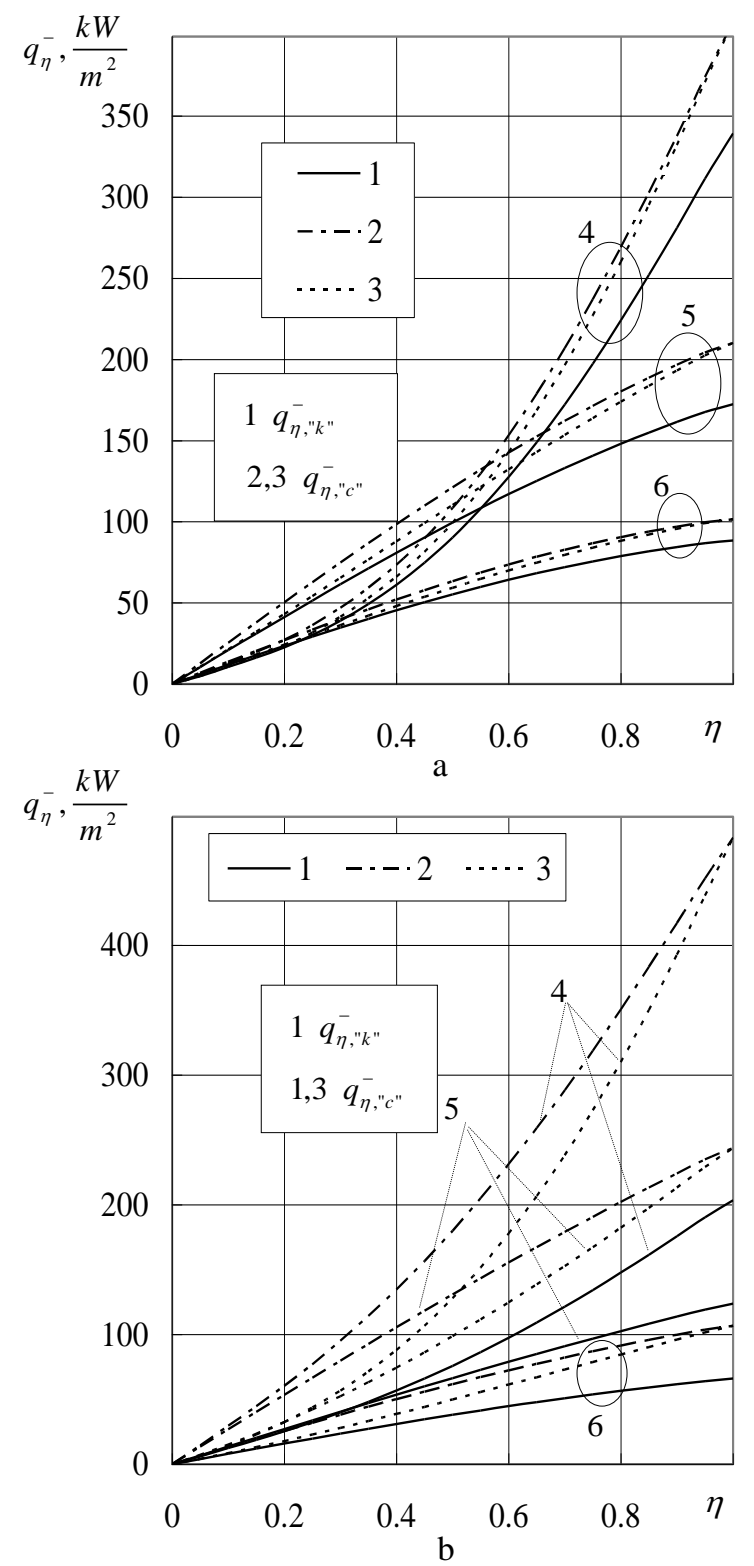

Fig. 5 Droplet slipping impact for heat abstraction intensity to central layers. a - $R e_{0}=5 ; \quad F o: 4-0.074$; $5-0.481 ; 6-0.703 ;$ b $-R_{0}=40 ;$ Fo: 4-0.115; $5-0.7475 ; 6-1.0925$. Heat spread model inside the droplet: (1) " $k$ ", (2) "c" strong circulation case, (3) " $c$ " liner receding circulation case

Calculated local heat flow spread in droplet depends from applied model of heat exchange in the droplet (Fig. 5). Heat transfer model influence is brightest in con- densation phase transformation regime, where droplet slipping is the most intensive.

Table 2

Internal convection heat flow density and its conductivity and convectional components, $\mathrm{kW} / \mathrm{m}^{2}$

\begin{tabular}{|c|c|c|c|c|c|c|}
\hline$R e_{0}$ & $q_{c, 0}^{-}$ & $q_{k, " c ", 0}^{-}$ & $q_{c, " c ", 0}^{-}$ & $q_{c, c o}^{-}$ & $q_{k, " c c^{\prime \prime}, c o}^{-}$ & $q_{c, " c ", c o}^{-}$ \\
\hline 0 & 388 & 388 & 0 & 62.7 & 62.70 & 0 \\
\hline 5 & 439.4 & 354.2 & 85.2 & 87.6 & 67.50 & 20.1 \\
\hline 10 & 460.2 & 249.5 & 210.7 & 92.8 & 55.90 & 36.9 \\
\hline 20 & 489.3 & 143 & 346.3 & 96.1 & 48 & 48.1 \\
\hline 40 & 530.2 & 96.2 & 434 & 100.2 & 40.9 & 59.3 \\
\hline 80 & 587.9 & 86.9 & 501 & 103.9 & 33.80 & 70.1 \\
\hline
\end{tabular}
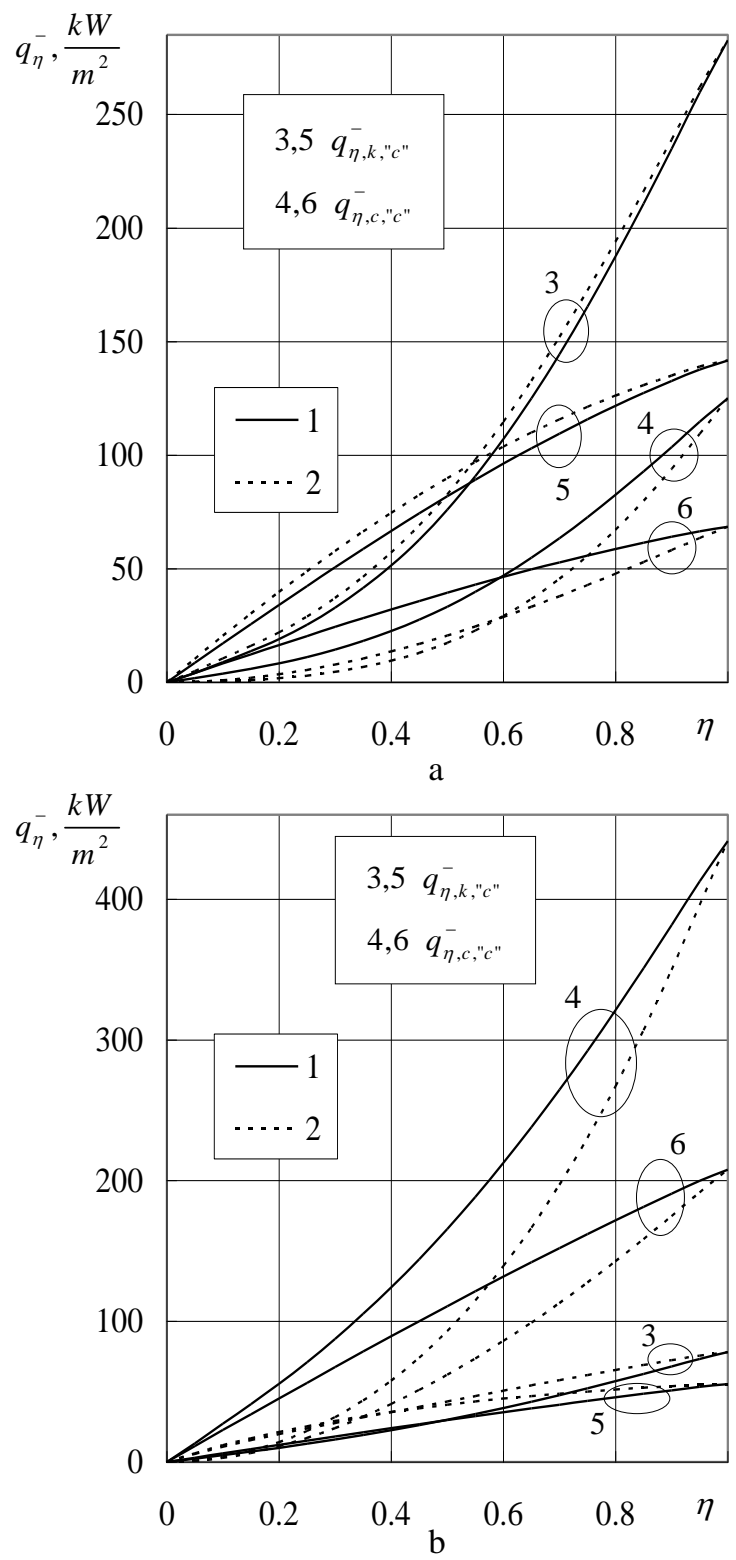

Fig. 6 Droplet slipping impact for convection heat flow components. a - $R e_{0}=5 ; \quad F_{O}: \quad 3,4-0.074$; $5,6-0.481 ; \quad$ b $-R e_{0}=80 ; \quad F o: \quad 3,4-0.115$; 5, $6-0.7475$. Heat spread model inside the droplet: (1) " $c$ " strong circulation case, (2) " $c$ " liner receding circulation case 
In all modelled slipping droplet cases, heat flow density $q_{\eta, "}{ }^{\prime \prime}, i$ calculated according to case,$k^{\prime \prime}$ is less than heat flow density calculated $q_{\eta,},{ }^{\prime}, i$ according to (7) expression. The difference between them is brighter at more intensive water circulation (Fig. 5, b). Strong and liner receding circulation in the droplet models for small slipping velocities gives close result (Fig. 5, a), while for larger slipping velocities a brighter difference already observes between calculated $q_{\eta,{ }^{\prime}{ }^{\prime}, i}$ heat flows (Fig. 5, b).

In slipping droplet the distribution between spreading local heat flow $q_{\eta,{ }^{\prime},{ }^{\prime}, i}$ convective $q_{\eta, c,},{ }^{\prime}, i$ and conductivity $q_{\eta, k},{ }^{\prime}{ }^{\prime}, i$ components depends from droplet slipping velocity (Fig. 6). At small slipping velocities conduction heat flow $q_{\eta, k,{ }^{\prime},{ }^{\prime}, i}$ is brighter in a droplet (Fig. 6, a). When slipping velocity increasing a heat flow spread convective component $q_{\eta, c, c}{ }^{,}, i$ influence also grows (Fig. 6, b).
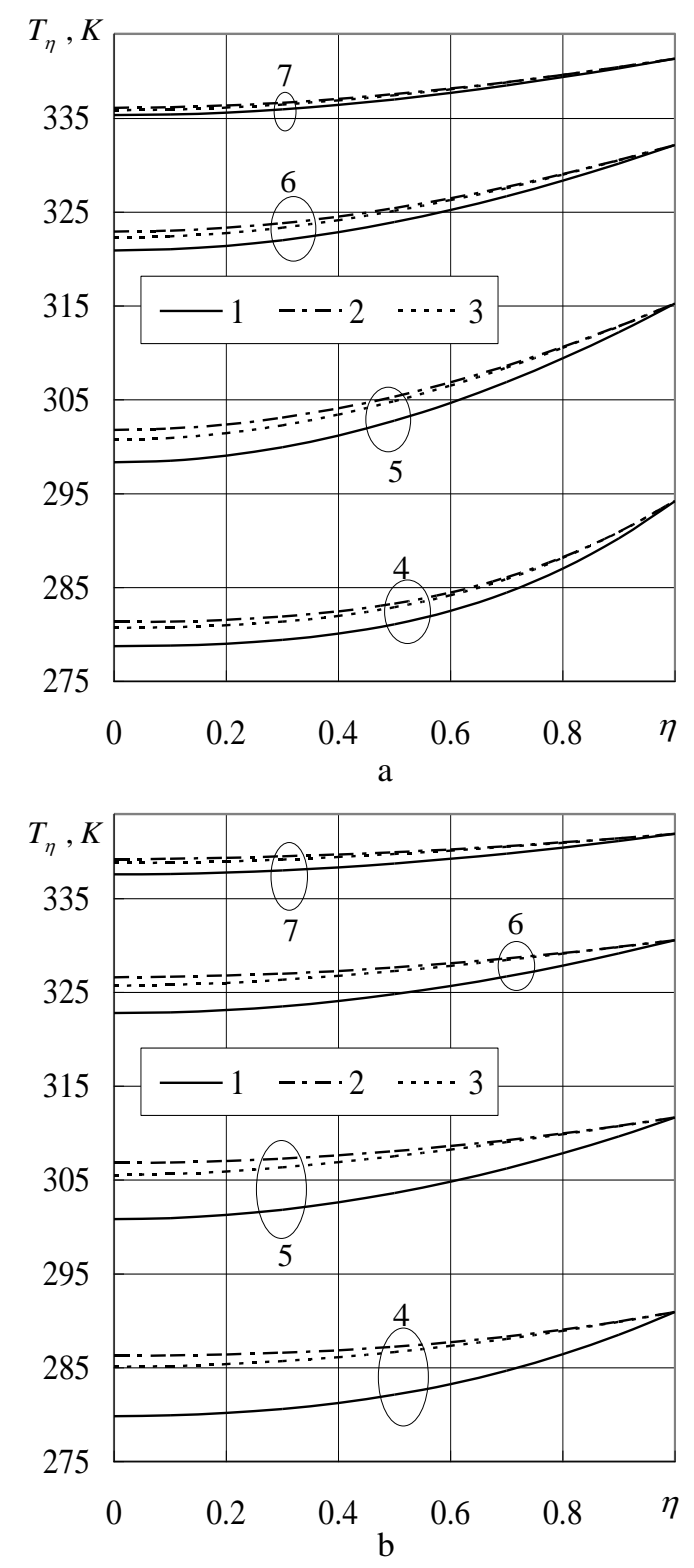

Fig. 7 Droplet slipping impact for local temperature. a $-R e_{0}=5 ; \quad F o: 4-0.074 ; 5-0.259 ; 6-0.481$; $7-0.703 ; \mathrm{b}-R e_{0}=40 ; F_{o}: 4-0.115 ; 5-0.4025$; $6-0.7475 ; 7-1.0925$. Heat spread model inside the droplet: (1) " $k ",(2)$ " $c$ " strong circulation case, (3) " $c$ " liner receding circulation case
In faster slipping droplet water circulates more intensively and heat is leaded to its central layers more quickly too (Fig. 5). Therefore droplet heats up equally and momentum temperature dissolution in " $c$ " heat transfer case is strong different from " $k$ " heat transfer case (Fig. 7). In strong circulation model case a calculated droplet temperature in central layers is lightly higher than in case of liner receding circulation model. It is observed in low (Fig. 7, a) and strong (Fig. 7, b) slipping droplets.
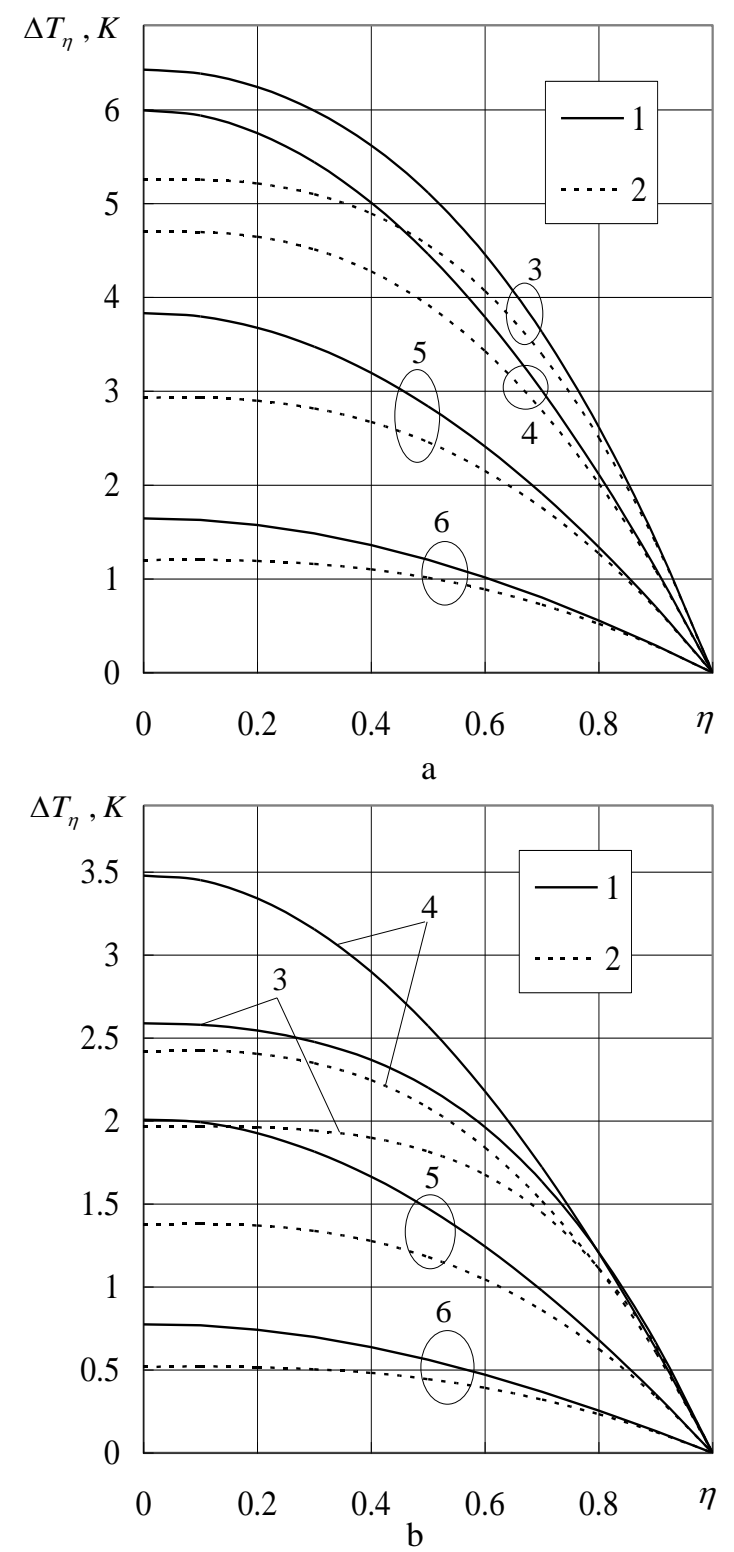

Fig. 8 Liquid circulation model inside the droplet impact for calculated local temperatures deviation from " $k$ "

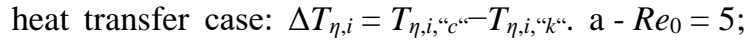
Fo: 3-0.074; 4-0.259; 5-0.481; 6-0.703; b $-R e_{0}=40 ; F o: 3-0.115 ; 4-0.4025 ; 5-0.7475$; $6-1.0925$. (1) "c" strong circulation, (2) " $c$ " liner receding circulation

At the initial stage of droplet heating the biggest difference observes between curves 2 and 3 that reflects instantaneous temperature field $T_{\eta, i}, " c$ " (Fig. 7). However this difference is less than one and half degrees and in unsteady phase transformation regime is consistently decreasing (Fig. 8). 
Maximum deviation of local temperature $T_{\eta, i,}$ " from conductivity heated droplet temperature $T_{\eta, i},{ }^{\prime}$ " , for slipping droplet reaches $3.5 \mathrm{~K}$ and $6.5 \mathrm{~K}$ when $R e_{0}=5$ and $R e_{0}=40$ in its central layers, respectively (Fig. 8).

A selected heat transfer model has influence for average droplet mass temperature which is calculated ac according to expression (1) (Fig. 9). Especially bright difference observes between droplet thermal modelling results in heat transfer cases of " $c$ " and " $k$ ". Strong and liner receding circulation models give close droplet mass average temperature dynamics (Fig. 9, 2 and 3 curves). For further slipping droplet analysis " $c$ " heat transfer case of strong circulation has been chosen.

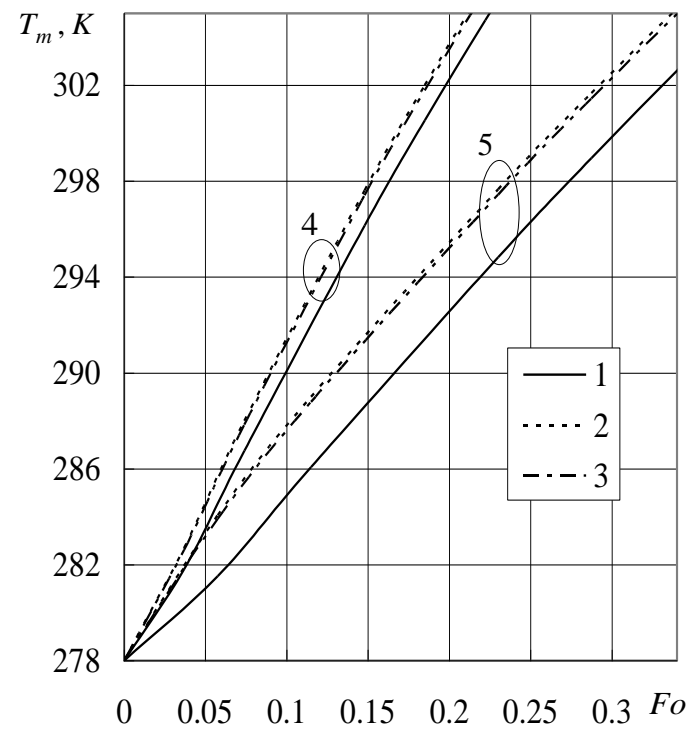

Fig. 9 Heat transfer model impact for average mass temperature of non-isothermal droplet: (1) " $k$ " heating, (2) " $c$ " strong circulation, (3) " $c$ " liner receding circulation case. $\operatorname{Re}_{0}: 4-5 ; 5-80$

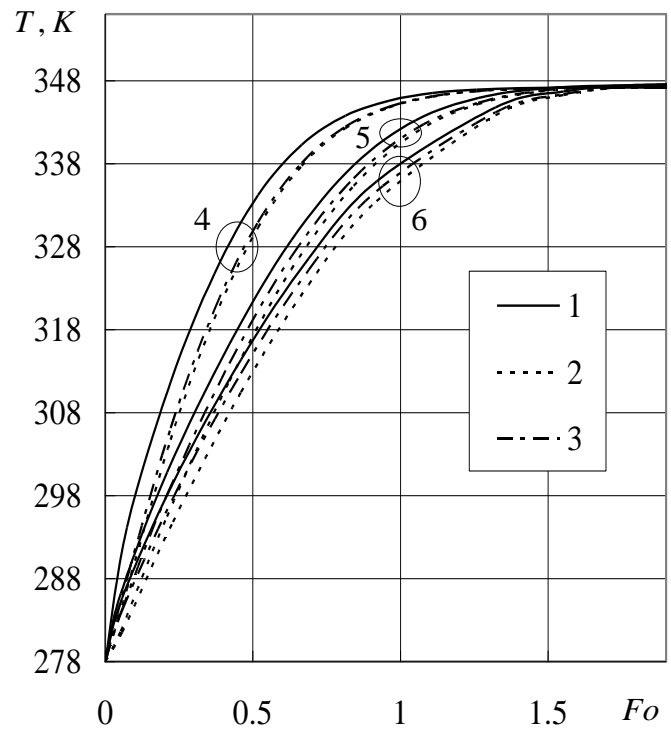

Fig. 10 Droplet slipping impact for calculated nonisothermal droplet thermal state: (1) $T_{R}(F o)$; (2) $T_{m}(F o)$ " $k$ " heat transfer case; (3) $T_{m}(F o)$ " $c$ " heat transfer case. $\operatorname{Re}_{0}: 4-5 ; 5-20 ; 6-80$
A calculated droplet surface temperature function $T_{R}(F o)$ graph do not depends from heat transfer model inside the droplet. However it is strongly affected by droplet slipping intensity in gas (Fig. 10). Droplet average mass temperature function $T_{m}(F o)$ graph that describes nonisothermal droplet thermal state change depends from applied heat transfer model for droplet and when droplet slips more intensively, this graph deviates from " $k$ " case graph (Fig. 10).

Influence of applied heat transfer model in droplet for calculated thermal state of droplet changes at time: it is significant in droplet condensing regime (Fig. 9), is weaken at unsteady evaporation regime and in equilibrium evaporation regime " $k$ " and " $c$ " heat transfer models ensures the same calculated droplet thermal state (Fig. 11).

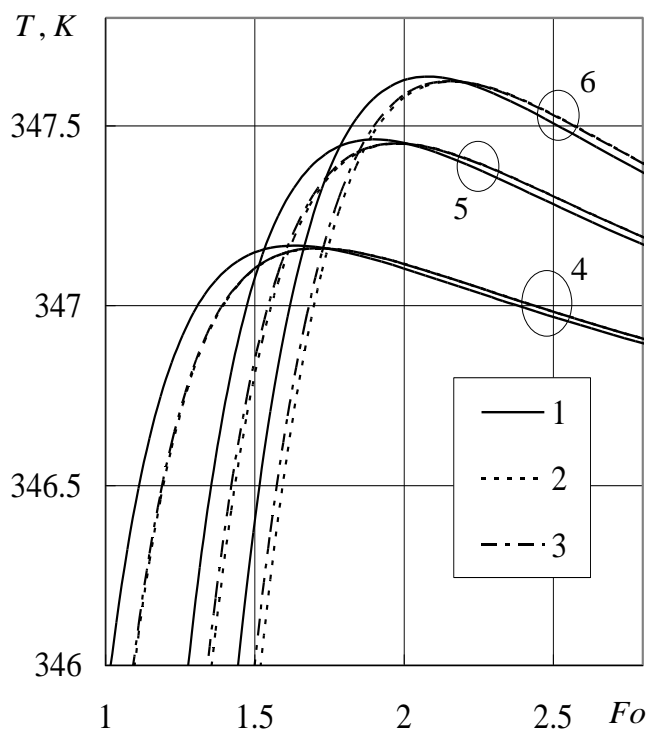

Fig. 11 Droplet surface and mass temperatures change in unsteady final stage and in initial stage of equilibrium evaporations: (1) $T_{R}(F o)$; (2) $T_{m}(F o)$ "k"; (3) $T_{m}(F o)$ " $c$ " heat transfer case. $\operatorname{Re}_{0}: 4-5 ; 5-20$; $6-80$

Table 3

Slipping impact for droplet layers temperature at the end of condensing regime

\begin{tabular}{|c|c|c|c|c|c|c|}
\hline$R e_{0}$ & $\tau_{c o}^{*}$ & $T_{\eta=0, c o}^{*}$ & $T_{\eta=5, c o}^{*}$ & $T_{\eta=.8, c o}^{*}$ & $T_{\eta=1, c o}^{*}$ & $T_{m}^{*}$ \\
\hline 0 & 0.0304 & 337.66 & 338.95 & 340.75 & 342.20 & 340.52 \\
\hline 5 & 0.0310 & 337.63 & 338.93 & 340.77 & 342.27 & 340.54 \\
\hline 10 & 0.0361 & 338.47 & 339.53 & 341.01 & 342.25 & 340.83 \\
\hline 20 & 0.0481 & 340.47 & 341.22 & 342.3 & 343.21 & 342.17 \\
\hline 40 & 0.0483 & 340.63 & 341.27 & 342.22 & 343.02 & 342.11 \\
\hline 80 & 0.0483 & 339.73 & 340.34 & 341.24 & 342.01 & 341.13 \\
\hline
\end{tabular}

* parameters are defined according to final iterative cycle results $i \equiv 21$ of condensing regime.

Droplet slipping has influence for calculated diameter dynamics (Fig. 12), therefore external convectional heat flow density that describes droplet energy state change in unsteady evaporation closing state is different (Fig. 13).

Water circulation inside the droplet ensures more 
intensive heat, that is provided to surface, abstraction to central layers and measurable operates droplet layers warming rate (Fig. 14). This impacts droplet unsteady temperature field dynamics (Fig. 7) and droplet layers warms differently (Table 3) in condensing phase transformation regime.

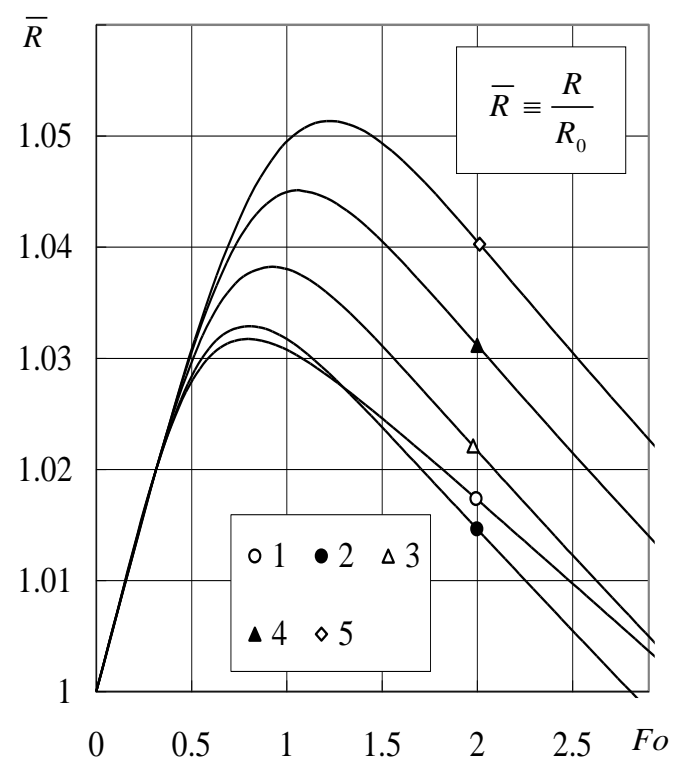

Fig. 12 Droplet diameter change in unsteady phase transformation regime and in initial stage of equilibrium evaporation. $R e_{0}:$ (1) 0 ; (2) 5 ; (3) 10 ; (4) 20 ; (5) 80

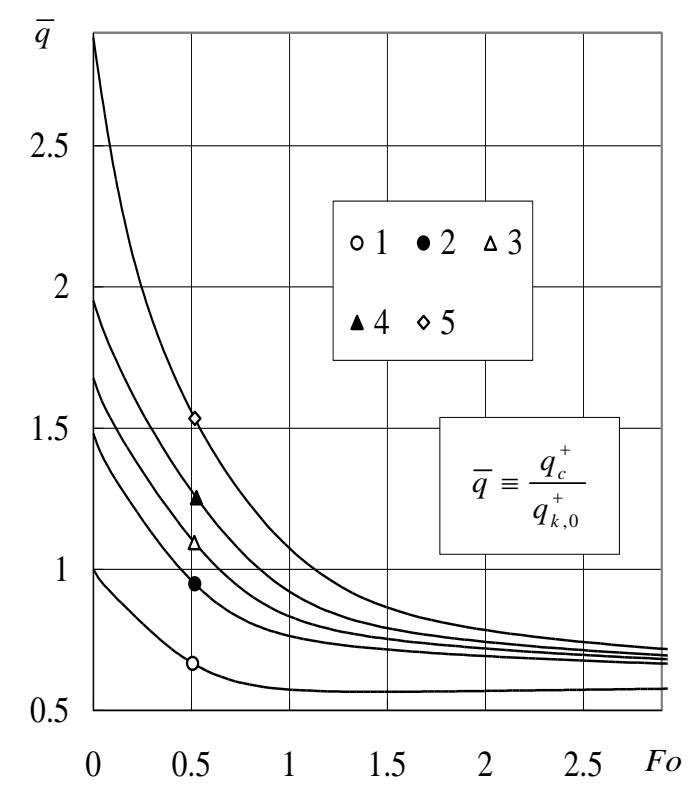

Fig. 13 Droplet convective heating intensity change in unsteady phase transformation regime and in initial stage of equilibrium evaporation. $R e_{0}$ : (1) 0 ; (2) 5 ; (3) 10 ; (4) 20 ; (5) 80

When primary slipping velocity in gas increases, then droplet layers average warming rate slowdown in condensing phase transformation regime (Table 4). This leads to duration increasing of condensing phase transformation regime.
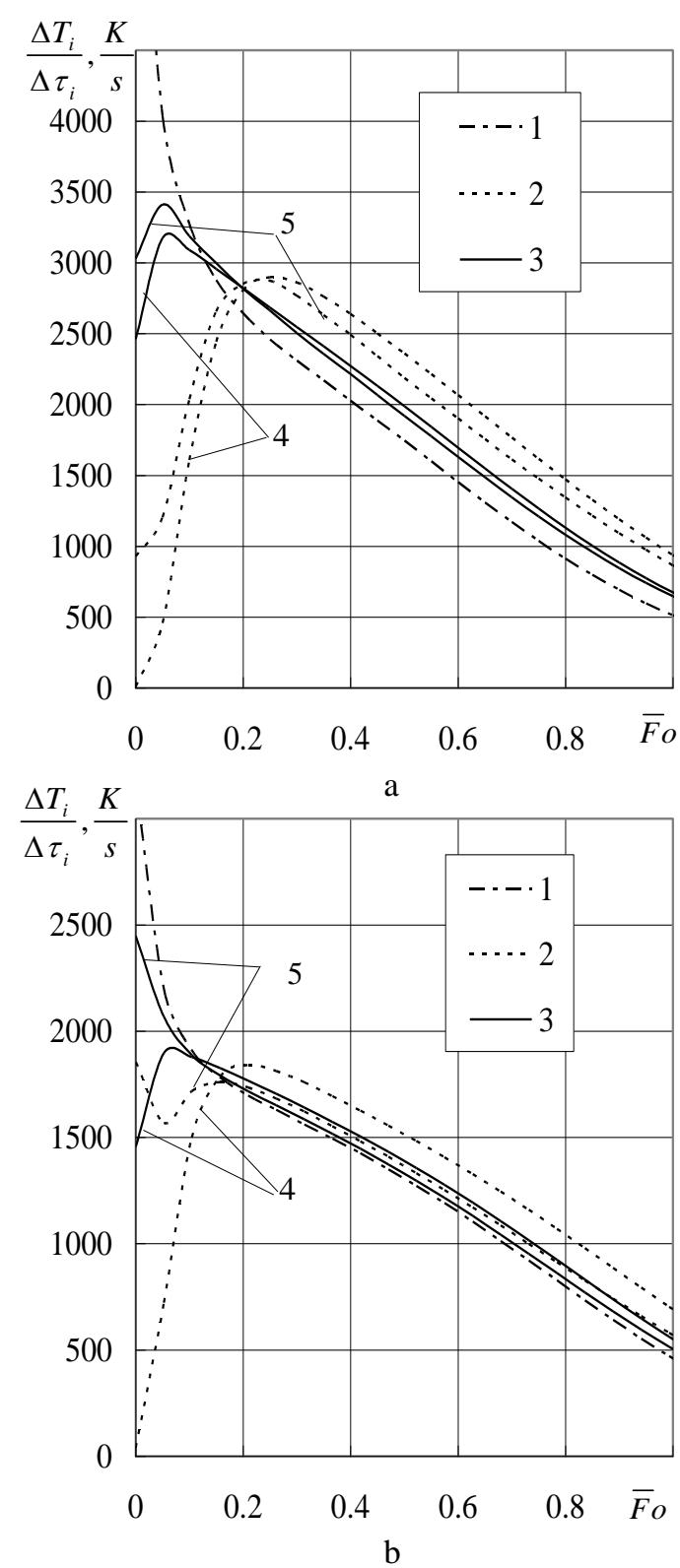

Fig. 14 Droplet slipping impact for its layers warming rate in condensing phase transformation regime $\Delta T_{i} / \Delta \tau_{i} \equiv\left(T_{j, i+1}-T_{j, i}\right) /\left(\tau_{i+1}-\tau_{i}\right)$, when $i<I$. $T_{\eta}$ : (1) $T_{\eta=1} \equiv T_{R}$; (2) $T_{\eta=1} \equiv T_{C}$; (3) $T_{\eta \equiv 0 \div 1} \equiv T_{m}$; heat transfer case: $4-" k " ; 5-" c "$; droplet primary slipping intensity: $\mathrm{a}-R e_{0}=5 ; \mathrm{b}-R e_{0}=40$

Table 4

Slipping impact for average warming rate $\Delta T_{\eta} / \Delta \tau_{c o} \equiv\left(T_{\eta, c o}-T_{0}\right) / \tau_{c o}$ of droplet layers at condensing regime

\begin{tabular}{|c|c|c|c|c|c|}
\hline$R e_{0}$ & $\frac{\Delta T_{\eta=0}^{*}}{\Delta \tau_{c o}^{*}}$ & $\frac{\Delta T_{\eta=.5}^{*}}{\Delta \tau_{c o}^{*}}$ & $\frac{\Delta T_{\eta=.8}^{*}}{\Delta \tau_{c o}^{*}}$ & $\frac{\Delta T_{\eta=1}^{*}}{\Delta \tau_{c o}^{*}}$ & $\frac{\Delta T_{m}^{*}}{\Delta \tau_{c o}^{*}}$ \\
\hline 0 & 1959.4 & 2001.7 & 2060.8 & 2108.4 & 2053.3 \\
\hline 5 & 1918.5 & 1960.4 & 2019.5 & 2068. & 2012.2 \\
\hline 10 & 1674.3 & 1703.1 & 1744.4 & 1778.9 & 1739.4 \\
\hline 20 & 1298.9 & 1314.6 & 1337.1 & 1356.1 & 1334.4 \\
\hline 40 & 1296.6 & 1310 & 1329.5 & 1346.1 & 1327.3 \\
\hline 80 & 1278 & 1290.7 & 1309.3 & 1325.3 & 1307.1 \\
\hline
\end{tabular}




\section{Conclusions}

Summarizing slipping droplet thermal state modelling results it can be state, that calculated droplet thermal state according to strong circulation model in weak slipping case is close to liner receding circulation modelling results, while for intensive slipping droplet case according to liner receding circulation model calculated droplet thermal state is bright different from strong circulation modelling results. Therefore in all droplets slipping case it is recommended to apply a strong circulation model.

Droplet slipping in humid air flow has an impact for complex heat transfer processes interaction in droplet condensing phase transformation regime and makes preconditions for intensive heat drain to inner layers with circulating water. Water warms evenly in slipping droplet then a surface layers warming rate slows down and condensing phase transformation regime duration increases. This is very important factor for technological processes optimization of heat phase transformation utilization from removable gas.

\section{References}

1. Sadafi, M.H.; Jahn, I.; Stilgoe, A.B.; Hooman, K. 2015. A theoretical model with experimental verification for heat and mass transfer of saline water droplets, Int. J. Heat Mass Transfer 81: 1-9.

http://dx.doi.org/10.1016/j.ijheatmasstransfer.2014.10.0 05 .

2. Wen-long, C.; Hua, C.; Lei, H.; Wei, Z. 2015. Effect of droplet flash evaporation on vacuum flash evaporation cooling: Modeling, Int. J. Heat Mass Transfer 84: 149-157.

http://dx.doi.org/10.1016/j.ijheatmasstransfer.2014.12.0 78.

3. Volkov, R.S.; Kuznetsov, G.V.; Strizhak, P.A. 2015. Experimental investigation of mixtures and foreign inclusions in water droplets influence on integral characteristics of their evaporation during motion through high-temperature gas area, International Journal of Thermal Sciences 88: 193-200. http://dx.doi.org/10.1016/j.ijthermalsci.2014.10.002.

4. Kyoung, H.K.; Hyung-Jong, K.; Kyoungjin, K.; Horacio, P.B. 2012. Analysis of water droplet evaporation in a gas turbine inlet fogging process, Applied Thermal Engineering 33-34: 62-69.

http://dx.doi:10.1016/j.applthermaleng.2011.09.012.

5. Sarkar, S.; Raghuram, S.; Vasudevan, R. 2013. Transient evaporation of moving water droplets in steamhydrogen-air environment, Int. J. Heat Mass Transfer 64: 536-546.

http://dx.doi.org/10.1016/j.ijheatmasstransfer.2013.04.0 66.

6. Tseng, C.C.; Viskanta, R. 2006. Enhancement of water droplet evaporation by radiation absorption, Fire Safety Journal 41: 236-247. http://dx.doi:10.1016/j.firesaf.2006.01.001.

7. Miliauskas, G. 2001. Regularities of unsteady radiative-conductive heat transfer in evaporating semitransparent liquid droplets, Int. J. Heat Mass Transfer 44: 785-798

PII: S00 1 7-93 10 ( 00$) 0012$ 7- 7.

8. Richard, W.B III. 2013. Correlation for drop wise condensation heat transfer: Water, organic fluids, and inclination, Int. J. Heat Mass Transfer 61: 1790-1803. http://dx.doi.org/10.1016/j.ijheatmasstransfer.2012.12.0 45.

9. Jun, G.; Chu, N.; William, H.M. 2012. Polydisperse aerosol condensation with heat and mass conservation: I. Model description with applications to homogeneous systems, Int. J. Heat Mass Transfer 55: 2429-2439. http://dx.doi:10.1016/j.ijheatmasstransfer.2012.01.017.

10. Miliauskas, G.; Šinkūnas, S.; Miliauskas, G. 2010. Eva-poration and condensing augmentation of water droplets in flue gas, Int. J. of Heat and Mass Transfer 53: 1220-1230.

http://dx.doi:10.1016/j.ijheatmasstransfer.2009.10.005.

11. Kortsensteyn, N.M.; Samuilov, E.V.; Yastrebov, A.K. 2009. About use of a method of direct numerical solution for simulation of bulk condensation of supersaturated vapor, Int. J. of Heat and Mass Transfer 55: 548556.

http://dx.doi:10.1016/j.ijheatmasstransfer.2008.06.033.

12. Roland, F.E.; Mirco, W.; Eugeny ,Y.K. 2014. The impact of Marangoni convection on fluid dynamics and mass transfer at deformable single rising droplets - A numerical study, Chemical Engineering Science 116: 208-222.

http://dx.http://dx.doi.org/10.1016/j.ces.2014.04.023.

13. Abramzon, B.; Sirignano ,W. A. 1989. Droplet vaporization model for spray combustion calculations, Int. J. of Heat and Mass Transfer 32: 1605-1618.

ftp://ftp.demec.ufpr.br/CFD/bibliografia/propulsao/abra mzon_et_al 1989.pdf. http://dx.doi.org/10.1016/0017-9310(89)90043-4.

G. Miliauskas, M. Maziukienè, A. Balčius, J.Gudzinskas

MODELLING OF HEAT AND MASS TRANSFER PROCESSES IN PHASE TRANSFORMATION CYCLE OF SPRAYED WATER INTO GAS:

4. THERMAL STATE ANALYSIS OF A DROPLET SLIPPING IN HUMID AIR FLOW

S u m m a r y

This article presents a heat transfer model that is based on by heat conductivity theory in slipping droplet. Strong and liner receding liquid circulation cases were analysed. According to received simulation results of thermal state by slipping droplet, it can be state that according to strong circulation model a calculated droplet thermal state in weak slipping case is close to liner receding modelling results, while a droplet thermal state is bright different from strong circulation modelling results for intensive droplet slipping case according to liner recieding circulation model. Therefore, it is recommended to apply a strong liquid circulation model for all droplet slipping cases.

Keywords: water droplets, convectional heating, phase transformation cycle, water circulation, thermal state.

Received October 02, 2015

Accepted March 15, 2016 